\title{
Metadata Use Type
}

National Cancer Institute

\section{Source}

National Cancer Institute. Metadata Use Type. NCI Thesaurus. Code C114465.

A description of the implementation of metadata. 